\title{
Power station based on the energy of the sea waves
}

\author{
Ratko Isidorovic $^{1}$, Janko Isidorovic ${ }^{2}$ and Nemanja Grubor ${ }^{1}$ \\ ${ }^{1}$ Minel Trafo AD \\ Kralja Petra I 332, 11400 Mladenovac, Serbia \\ ${ }^{2}$ Pere Segedinca 7, 11070 Novi Beograd, Serbia \\ phone:+381 11172 607, e-mail: jankoi@sezampro.yu
}

phone:+381 118231 234, fax:+381 118232 720, e-mail: Isidorovic.Ratko@minel.co.yu, nemanja@minel.co.yu

\begin{abstract}
In this paper is shown working principal of wave pump, which simultaneously draws energy from larger number of sea waves. The pump could pump the water, but also the air. It is possible to form particular systems, wave electric power stations, made of larger number of wave pumps. Simplified calculation shows that there is significant energy in question. Simultaneous drawing energy from sea waves, gives the possibility to collect energy from larger to smaller space, for example space of turbine with generator of electric energy.

Wave electric power stations, if near the coast, could pump the water and form water accumulation on the land. Potential energy of these accumulations could be used in intervals when there are no waves on the sea.

If wave electric power stations are on the ocean, far away from the coast, they could produce hydrogen. Hydrogen will be transported to the land and used as power fuel.

Described wave electric power station satisfies principal of physics, but it does not mean that it is technically feasible due to moods of seas and oceans.
\end{abstract}

Keywords: Sea, wave, pump, power station, energy

\section{General}

In this paper is explained the possibility of constructing a large number of wave pumps, which draw energy from waves of Adriatic Sea. Energetic abilities of the waves of Adriatic Sea as well as of Atlantic Ocean, have been analyzed in general.

\section{Description}

Wave pump working principle can be described by Fig.1. Vertical movement of the changeable direction of water molecules, i.e. transversal wave is marked as W. Such waves are formed at seas and oceans due to blowing winds energy, i.e. energy of winds is transformed into sea waves energy. If the float is placed on a sea surface it will oscillate up and down, but it will stay at one point, i.e. it will always cut the horizontal axis at the same point, although the waves are moving. Fig.1 shows several floats $\mathrm{p}_{1}, \mathrm{p}_{2} \ldots \ldots \mathrm{p}_{\mathrm{n}}$, firmly connected to levers $\mathrm{n}$ of the equal lengths and particulary shaped. The levers $n$ are firmly connected to levers $m$ under angles $\alpha_{1} \ldots \ldots \ldots \alpha_{n}$. At the spot where levers $\mathrm{m}$ and $\mathrm{n}$ are firmly interconnected there is an axle $\mathrm{O}_{1}$ connected to a rigid stick q. Axels $\mathrm{O}_{1}$ are placed at identical distances 1 . At the other end of the levers $\mathrm{n}$ are placed axles $\mathrm{O}_{2}$ linked to an elastic hose $\mathrm{C}$. The length of the hose 1 between the axles $\mathrm{O}_{2}$ is always the same, but it is elastic (like accordion bellow), so its length 1 can easily be increased or decreased along the longitudinal axis. Valves $t_{1}, t_{2} \ldots . . t_{n}$ are placed at the spots where axle $\mathrm{O}_{2}$ is linked to the hose. Valves are opened by pressure in $\mathrm{V}_{3}$ direction, and are closed by pressure in the opposite direction.

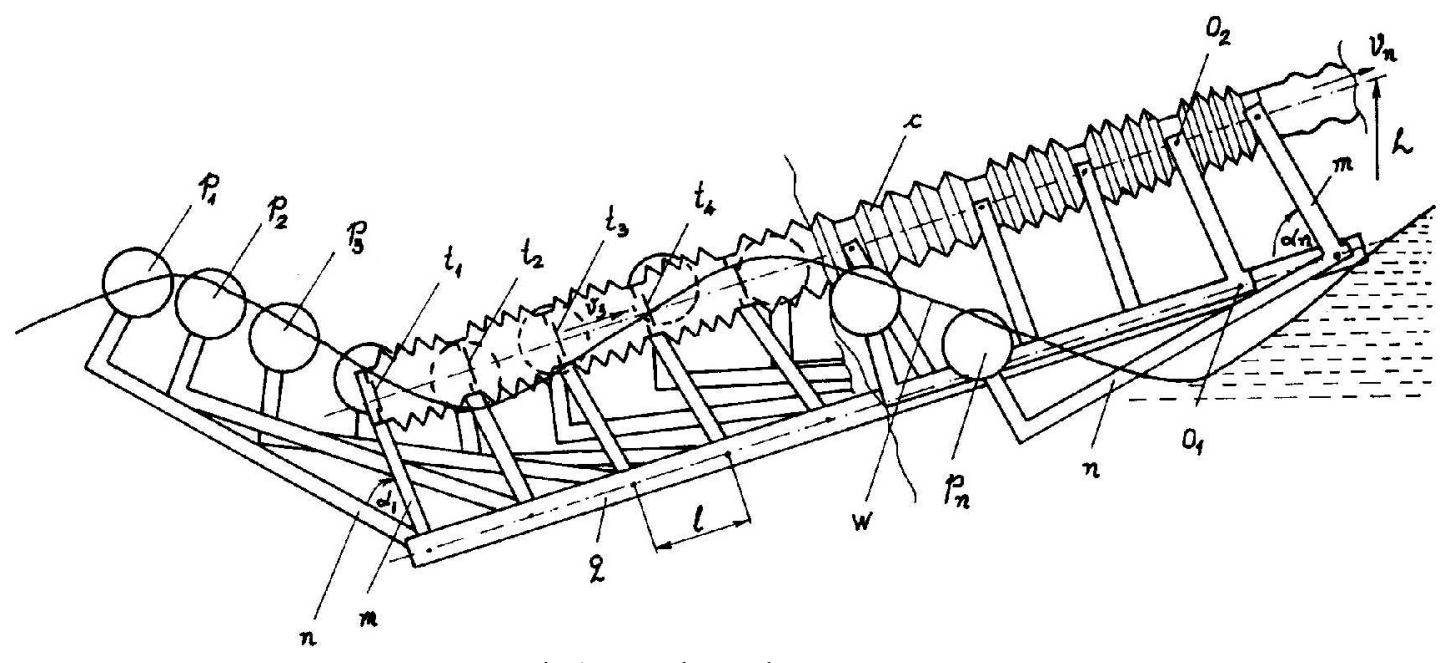

Fig 1. Experimental wave pump 
The hose, on the Fig.1, is placed under angle to the sea surface, so one end of hose is under sea surface and the other is on the height $h$. If the float $p_{1}$ is at the foot of the wave (point between two maximal points of the waves), the hose will maximally stretch between valves $t_{1}$ and $t_{2}$

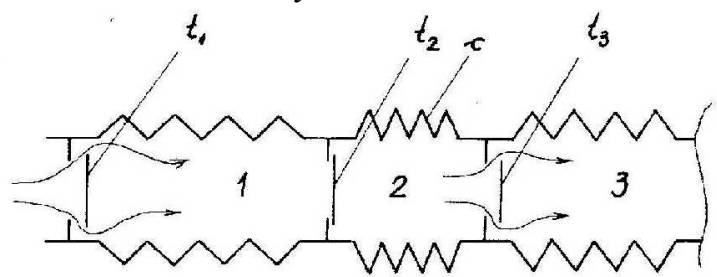

Fig 2. Entering water in volume 1 and crossing water from volume 2 in volume 3

When the float $\mathrm{p}_{1}$ begins to climb up the wave slope, the hose between valves $t_{1}$ and $t_{2}$ will shrink and reduce its volume, so that the pressure increase and it will now close valve $t_{1}$ and open valve $t_{2}$. It can easily be opened, as its float $p_{2}$ is on its way to the wave foot (wave minimum) so that the pressure decreases in the hose between valves $t_{2}$ and $t_{3}$ - Fig.3. Water will flow from volume 1 into volume 2 , as the volume of hose between valves $t_{1}$ and $t_{2}$ is decreasing and volume of the hose between valves $t_{2}$ and $t_{3}$ is increasing. This will proceed until float $\mathrm{p}_{1}$ reaches the top (wave maximum) and than the whole process repeats. Float $\mathrm{p}_{1}$ descends along the wave slope, volume 1 increases again and the water is absorbed again, float $\mathrm{p}_{2}$ climbs up the wave slope, volume 2 decreases and the water is pressed through valve $t_{3}$ into volume 3 etc. Thus, each passing of the waves over all floats will press the water, at the speed $V_{n}$, through the other end of the elastic hose, which is placed on the height $h$. At the hose end water will posses both potential and kinetic energy. One can choose. By changing the angles $\alpha$ the pump can be placed into horizontal position, so there will be no height potential energy but an increased pressure will throw out the water at higher speed $\mathrm{V}_{\mathrm{n}}$. Sea waves are variable values, but from Fig. 1 it can be observed that, for sinusoidal wave, one half of all the floats is always in the ascending phase up the wave slope, i.e. at the same time pressing the water into elastic hose. The result of this simultaneous action is that the highest pressure is at the end of the and will increase its volume. During this stretching the pressure between valves $t_{1}$ and $t_{2}$ will decreases valve $t_{2}$ will close and valve $t_{1}$ will open. As valve $t_{1}$ is always under sea surface, water will be absorbed in the hose as shown on Fig.2.

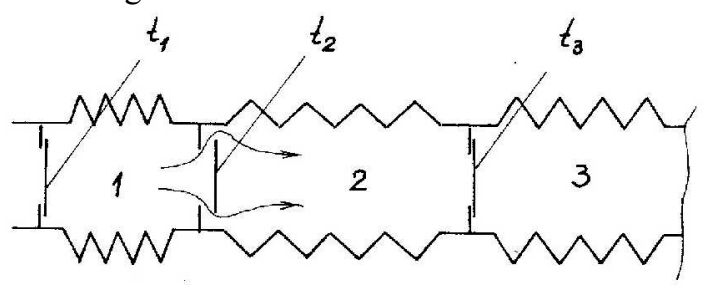

Fig 3. Crossing water from volume 1 in volume 2

hose. So it comes out that the energies of all floats are subtracted at the end of elastic hose, where can be placed turbine $\mathrm{y}$ with generator $\mathrm{g}$ for producing electric energy, as shown on Fig.4.

A number of pumps $t_{p 1}, t_{p 2}, t_{p 3} \ldots$. can be connected to one turbine. It should be noted that if a wave pump is of large lenght, i.e. if there is a large number of floats, huge pressure will appear at the end of elastic hose, so that resistance of incorporated material will be a limiting factor.

A large sea surface can be covered by such wave pumps and thus obtaining larger amount of energy. In order to estimate that energy, wave characteristics must be known.

The calculation of wave energy is based on the characteristics of the waves.

Each sea and ocean has its own characteristcs. Adriatic sea waves characteristics are shown in Table 1. Energy of the waves consists of kinetic and potential energy. Kinetic energy originates from circular movement of particles which extends to one half of the wave length into debth, Fig.5, and potential energy originates from position of particles related to calm sea surface. Developed expression for total energy is:

$$
E=\rho \cdot g \frac{\lambda \cdot \delta^{2}}{8} \cdot\left[1+\frac{4 \cdot \pi \cdot H / 4}{s \cdot h \cdot 4 \cdot \pi \cdot H / 4}\right]
$$

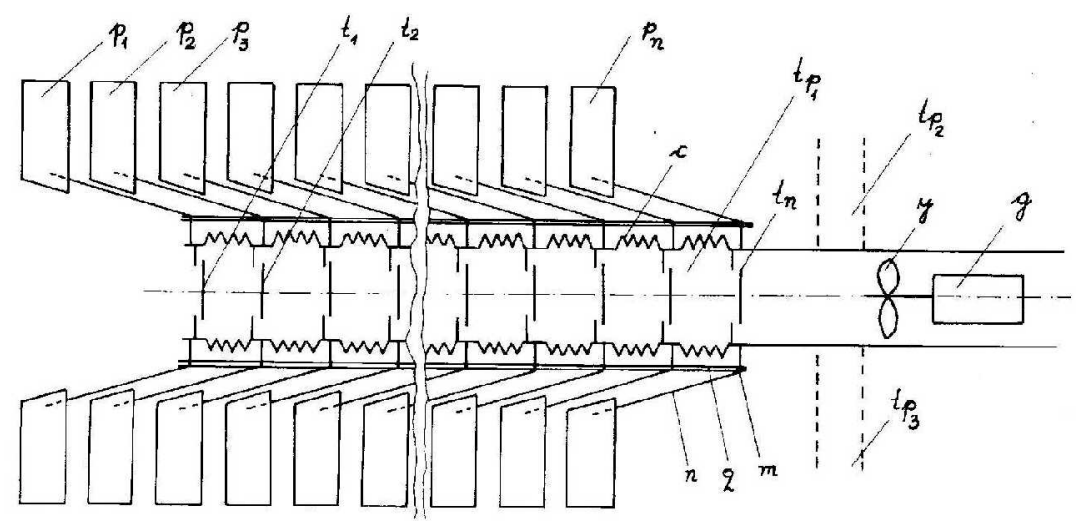

Fig. 4. Wave pump with turbine and generator 


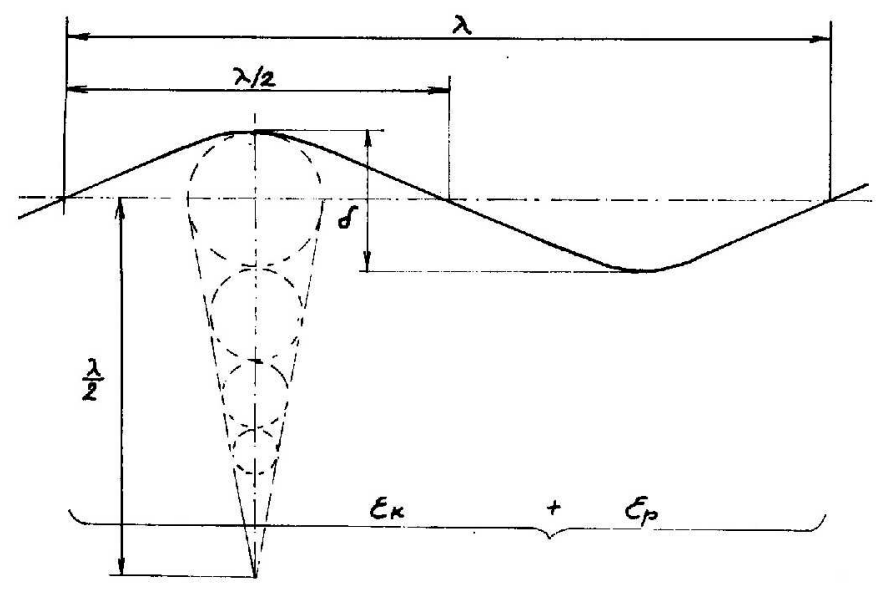

Fig. 5. Sinuous wave

The power will be obtained by dividing of energy by time. But in Table 1 energy is given in function of longitudinal meter of wave front $(\mathrm{kW} / \mathrm{m})$. It is considered that future electric power stations will be linearly dependent of the coast length, no matter which type will be applied later. Any electric power station, bound to the coast, can not receive more energy. Location of electric power station is very important, due to the fact that it receives only the waves directed to the coast. This points out very poor possibilities of consumption of waves energy.
However, the results are different if the wave pump is placed further from the Adriatic coast, as shown on Fig.6. Float dimensions are $(40 \mathrm{dm} \times 20 \mathrm{dm} x 4 \mathrm{dm})$, those dimensions are adopted, but optimal float dimensions should be explored in future. Wave characteristics given on Fig. 6 are: wave height $2.6 \mathrm{~m}$, wave length $32 \mathrm{~m}$ and period $6.9 \mathrm{sec}$. Suppose that wave pump contains 700 floats, 350 at each side, and if its length is $1000 \mathrm{~m}$, distance between two floats is $0.85 \mathrm{~m}$. The volume of one float is $40 \mathrm{dm} \times 20 \mathrm{dm} \times 4 \mathrm{dm}=3200 \mathrm{dm}^{3}$, so each float acts with power of: $\mathrm{F}=3200 \times 9.8=31360 \mathrm{~N}$

Table 1

\begin{tabular}{|c|c|c|c|c|c|}
\hline $\begin{array}{c}\text { BEUFORT'S } \\
\text { SCALE }\end{array}$ & Wind & \multicolumn{3}{|c|}{ Adriatic wave } & Wave power \\
& $\begin{array}{c}\mathrm{W} \\
\text { Speed } \\
\mathrm{m} / \mathrm{s}\end{array}$ & $\begin{array}{c}\delta \\
\text { Altitude } \\
\mathrm{m}\end{array}$ & $\begin{array}{c}\lambda \\
\text { Lenght } \\
\mathrm{m}\end{array}$ & $\begin{array}{c}\tau \\
\text { Period } \\
\mathrm{s}\end{array}$ & $\mathrm{kW} / \mathrm{m}$ \\
\hline 0.Silence & $0-0.2$ & 0 & 0 & 0 & 0 \\
\hline 1.Zephyr & $0.3-1.5$ & 0.05 & 2.0 & 1.6 & 0.0027 \\
\hline 2.Air & $1.6-3.3$ & 0.20 & 5.0 & 2.7 & 0.086 \\
\hline 3.Breeze & $3.4-5.4$ & 0.50 & 9.5 & 3.7 & 0.657 \\
\hline 4.Medium wind & $5.5-7.9$ & 0.80 & 14.0 & 4.6 & 2.767 \\
\hline 5.Fresh wind & $8.0-10.7$ & 1.30 & 20.0 & 5.4 & 8.446 \\
\hline 6.Medium strong wind & $10.8-13.8$ & 1.9 & 25.0 & 6.2 & 21.02 \\
\hline 7.Strong wind & $13.9-17.1$ & 2.6 & 32.0 & 6.9 & 45.42 \\
\hline 8.Very strong wind & $17.2-20.7$ & 3.5 & 39.0 & 7.6 & 88.56 \\
\hline 9.Stormy wind & $20.8-24.4$ & 4.6 & 46.5 & 8.3 & 160.0 \\
\hline 10.Strong storm & $24.5-28.4$ & 5.9 & 55.0 & 9.0 & 270.3 \\
\hline 11.Very strong storm & $28.5-32.6$ & 7.3 & 66.0 & 9.7 & 453.3 \\
\hline 12.Hurricane storm & $32.7-36.9$ & 8.8 & 79.0 & 10.4 & 673 \\
\hline
\end{tabular}




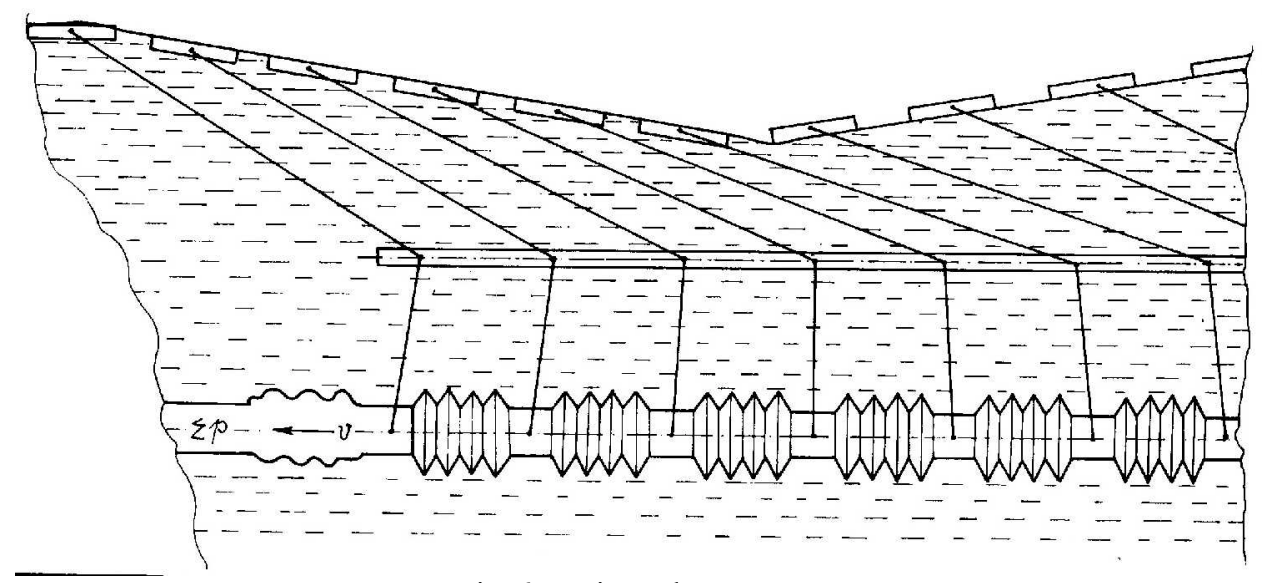

Fig. 6. Horizontal wave pump

Waves will rise simultaneously $700 / 2=350$ floats, so total power is: $F=31360 \times 350=10.976 .000 \mathrm{~N}$

The way that the float has to pass is equal to the wave height lessened for the float height, i.e. $\mathrm{s}=2.6-0.4=2.2 \mathrm{~m}$, and it will do it for the half of period $\mathrm{t}=6.9 / 2=3.45 \mathrm{sec}$, so float speed is $v=2.2 / 3.45=0.637 \mathrm{~m} / \mathrm{sec}$.

Total pump power is:

$\mathrm{P}=\mathrm{F} \times \mathrm{v}=$

$=10.976 .000 \times 0.637=6.991 .712 \mathrm{Nm} / \mathrm{sec}=6.99 \mathrm{MW}$
This example shows that, whenever there are waves, there is significant absorption of energy, and $2.5 \mathrm{~m}$ high waves on the Adriatic Sea are not rare as shown on climate atlas. Fig.7 shows the rose of the waves near island Mljet in January. Average and maximum heights of wave, in meters, are given at the end of each direction and time frequency is shown by line length. Stillstand without waves is written in the middle of the circle, as percent. Number of measurements are marked by separate number in lower part, above the line.

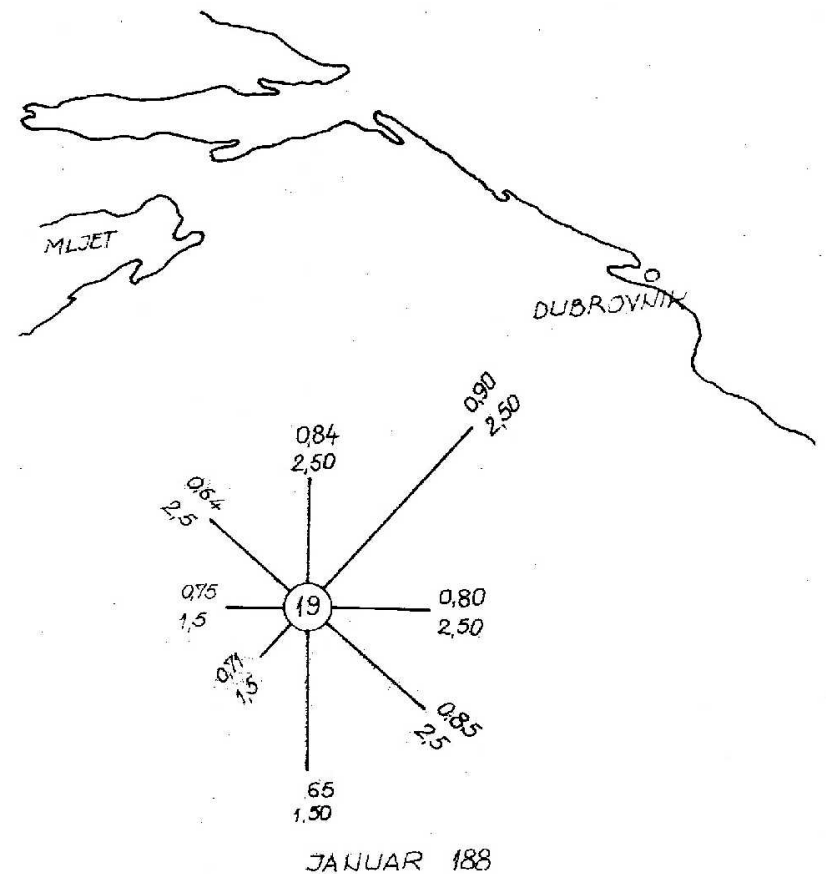

Fig. 7. Rose wave

Medium height of Adriatic sea waves is $0.8 \mathrm{~m}$,wavelength is $14 \mathrm{~m}$ and period $4.6 \mathrm{sec}$, so the energy obtained by pump described in the former example, can be calculated. The distance that float has to pass is:

$\mathrm{s}=0.8-0.4=0.4 \mathrm{~m}$.

Float speed is:

$v=\frac{s}{\tau / 2}=\frac{0.4}{2.3}=0.174 \mathrm{~m} / \mathrm{s}$
As the total force is known from the former example, pump power is:

$\mathrm{P}=\mathrm{F} \times \mathrm{v}=10.976 .000 \times 0.174=1.909 .824 \mathrm{Nm} / \mathrm{sec}=1.9 \mathrm{MW}$. Calculated powers ratio is $6.99 / 1.9=3.67$ and ratio of wave powers, from the Table 1 is $45.42 / 2.767=16,4$. It comes out that pump should be constructed in such a way to absorb proportionally the most energy from the average wave, as they are the most frequent. With the increase of the waves their energy increases too, but not proportionally, which is favorable, as by hurricane storms, the waves do not burden the pump construction with their full power. 
It has already been mentioned that the pump is placed further from the coast, let us say at the spot of wave rose given on Fig.7, so the waves are coming from all directions. As each of them rises and lowers the float, it comes out that waves of all directions and their energy are used. The energies of waves, after passing the pump floats, are decreased for the amount absorbed by floats. They do not break against it, as on the coast, but they still exist. The waves originate from the wind, and the wind still blows, it is sure that the waves chased by wind, after certain time, would regain the lost energy. Now, new pump can be installed and the process repeated again, etc. On larger seas and oceans the possibilities are greater. At same wind speed the waves are much higher. Average wave at Adriatic sea is formed under wind speed of $\mathrm{W}=6.7 \mathrm{~m} / \mathrm{sec}$, and for the same wind speed the wave formed on Atlantic ocean will be with following characteristics:

$\delta=0,405 \mathrm{~W}^{1.025}=2,84 \mathrm{~m}$

$\lambda=4,05 \mathrm{~W}^{1.3}=48 \mathrm{~m}$

$\tau=1,52 \mathrm{~W}^{0.665}=5,38 \mathrm{sec}$

If for such a wave the same pump is used and same calculation applied, obtained power at one pump would be $9.87 \mathrm{MW}$, what is 5.19 times more than of Adriatic sea. A system of 2400 pumps of the Atlantic ocean will have power capacity of $2400 \times 9.87=23688 \mathrm{MW}$, which by degree of only $20 \%$ exploitation would be equivalent to 4.7 nuclear power stations rated $1000 \mathrm{MW}$.

\section{Conclusion}

Described wave electric power station satisfies principals of physics, but it does not mean that it is technically feasible due to moods of seas and oceans.

Isolated pumps may not be more suitable, may be it is better to construct systems as spider web, Fig.8, or frontal along the shore, Fig.9. Perhaps, install complete construction on sea surface and pump the air by waves power. On the oceans, far away from coast hydrogen could be produced, transported to shore and used as power fuel.

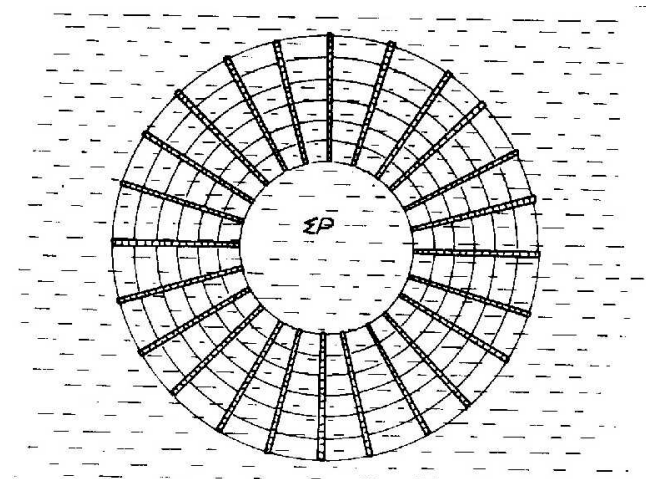

Fig. 8. System wave pump - spider web

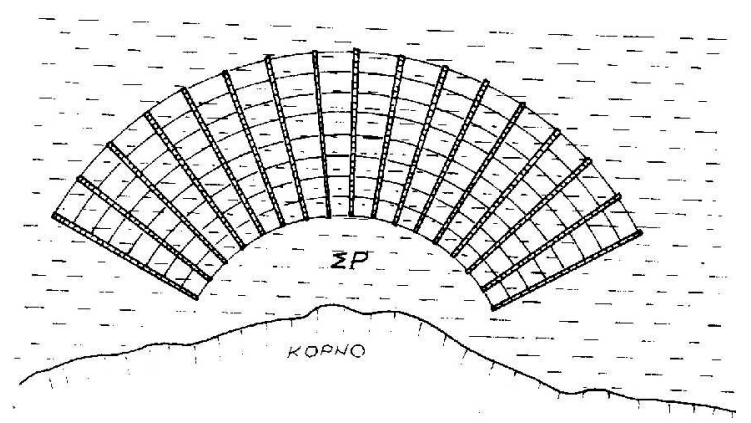

Fig. 9. Frontal system wave pumps

\section{References}

[1] Alka Mihelic-Bogdanovic, Rajka Budin, Energetski parametri valova Jadrana i Atlantika, Savetovanje o energiji zbornik referata, Opatija 1986.

[2] Ivo Kolin, Energija morskih valova, Energija i razvoj, Jugoslovenska naucna tribina, Beograd 1986. 